\title{
A Quantitative Model of ERK MAP Kinase Phosphorylation in Crowded Media
}

\section{SUBJECT AREAS: SYSTEMS BIOLOGY \\ BIOPHYSICS \\ CELL SIGNALLING \\ CELL GROWTH}

Received

30 January 2013

Accepted

8 March 2013

Published

26 March 2013

Correspondence and requests for materials should be addressed to K.A. (k-aoki@lif.kyotou.ac.jp)

\author{
Kazuhiro Aoki ${ }^{1,2}$, Koichi Takahashi ${ }^{3,4}$, Kazunari Kaizu $^{3}$ \& Michiyuki Matsuda ${ }^{1,5}$
}

'Laboratory of Bioimaging and Cell Signaling, Graduate School of Biostudies, Kyoto University, Sakyo-ku, Kyoto 606-8501, Japan, ${ }^{2}$ PRESTO, Japan Science and Technology Agency (JST), 4-1-8 Honcho Kawaguchi, Saitama 332-00 12, Japan, ${ }^{3}$ Laboratory for Biochemical Simulation, RIKEN Quantitative Biology Center, 6-2-3 Furuedai, Suita, Osaka, 565-0874, Japan, ${ }^{4}$ Institute for Advanced Biosciences, Keio University, 5322 Endo, Fujisawa, 252-0882, Japan, ${ }^{5}$ Department of Pathology and Biology of Diseases, Graduate School of Medicine, Kyoto University, Sakyo-ku, Kyoto 606-8501, Japan.

Cytoplasm contains a large number of macromolecules at extremely high densities. How this striking nature of intracellular milieu called macromolecular crowding affects intracellular signaling remains uncharacterized. Here, we examined the effect of macromolecular crowding on ERK MAPK phosphorylation by MEK MAPKK. Addition of polyethylene glycol-6000 (PEG-6000) as a crowder to mimic intracellular environments, elicited a biphasic response to the overall ERK phosphorylation rate.

Furthermore, probability of processive phosphorylation (processivity) of tyrosine and threonine residues within the activation loop on ERK increased non-linearly for increasing PEG-6000 concentration. Based on the experimental data, we developed for the first time a mathematical model integrating all of the effects of thermodynamic activity, viscosity, and processivity in crowded media, and found that ERK phosphorylation is transition-state-limited reaction. The mathematical model allows accurate estimation of the effects of macromolecular crowding on a wide range of reaction kinetics, from transition-state-limited to diffusion-limited reactions.

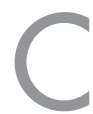
ells sense a variety of extracellular signals by receptors, and input signals to a system of intracellular reaction network, i.e., an intracellular signal transduction cascade. These input signals are processed by the intracellular signal transduction cascades to drive the cells to exhibit specific phenotypes. A collapse of the system by gene mutations leads to pathological outcomes, including autoimmune disease and cancer ${ }^{1}$. Therefore, a better understanding of the intracellular signal transduction cascade is one of the critical issues for the control of diseases. The intracellular signal transduction cascade consists of a chain of diffusion, protein-protein interaction and enzymatic reactions, and thus it can be described by ordinary and partial differential equations, and solved numerically in order to analyze its dynamics ${ }^{2-4}$. Such a systems biology approach with experimentally-verified kinetic parameters provides a basis for understanding the signal transduction cascade ${ }^{5-8}$.

The cytoplasm is highly crowded with macromolecules, such as proteins, lipids, nucleic acids and so on ${ }^{9,10}$. The concentration of proteins in the cytoplasm is over $100 \mathrm{mg} / \mathrm{ml}$, with macromolecules making up $30-40 \%$ of the cytoplasmic volume ${ }^{11,12}$. This congested condition, often referred to as macromolecular crowding, results in enzymatic reaction kinetics that differ significantly from those described previously in ideal-dilute solution. For example, macromolecular crowding affects the enzymatic reaction rate through two factors: thermodynamic activity and viscosity ${ }^{13-16}$. The former factor, thermodynamic activity, compensates for the concentration in an ideal solution to yield an effective concentration in a real solution ${ }^{15,17}$. Macromolecular crowding increases thermodynamic activity mainly through the excluded volume effect, and consequently leads to an increase in a reaction rate. The latter factor, viscosity, reduces an encounter rate between the enzyme and the substrate ${ }^{15,18}$. Therefore, the reaction rate is negatively correlated with viscosity.

Processive reactions can be observed in phosphorylation ${ }^{19,20}$, polyubiquitination ${ }^{21}$, and so on. These multisite posttranslational modifications of signaling proteins are a common mechanism by which a complex cellular phenotype can be exhibited ${ }^{22}$. We demonstrated by a computer simulation that crowding environment increased the processivity in two-step phosphorylation reactions of ERK MAP kinase ${ }^{23}$. This prediction was validated by our experimental results ${ }^{24}$.

Although macromolecular crowding has been suggested to have a great influence on the intracellular signaling pathway, it is not fully understood how the macromolecular crowding affects the overall reaction rate and processivity of enzymes. Here, we propose mathematical models of reaction kinetics that account for the effect 
of thermodynamic activity, viscosity and processivity in an environment with macromolecular crowding, and provide empirical validations by employing in vitro ERK MAP kinase phosphorylation.

\section{Results}

Quasi-processive phosphorylation of ERK under macromolecular crowding. We have recently shown that Tyr and Thr residues within the activation loop of ERK MAP kinase were phosphorylated by MEK in a quasi-processive manner in mammalian cells ${ }^{24}$. In the first step, MEK binds to and phosphorylates ERK to generate tyrosine monophosphorylated ERK ( $\mathrm{pY}$-ERK), and then dissociates from pY-ERK (Fig. 1A). Under macromolecular crowding, diffusion of MEK and pY-ERK is restricted. Therefore, rebinding of the MEK and the pY-ERK, which will not substantially happen in ideal solutes, occurs with some probability, and consequently the MEK processively phosphorylates pY-ERK, producing the tyrosine and threonine bisphosphorylated ERK (pTpY-ERK).

We analyzed the effects of macromolecular crowding by simplified quasi-processive phosphorylation model as follows. First, the rate constants of two successive phosphorylation reactions of ERK in the absence of a crowder agent were defined as $k_{1}$ and $k_{2}$, respectively (Fig. 1B, left). Our previous analysis demonstrated that these rate constants were first-order parameters, since the Michaelis constants in these reactions were significantly higher than the concentration of substrate, np-ERK, used in this study ${ }^{24}$. Under this condition, the $k_{1}$ and $k_{2}$ values can be determined by fitting experimental data (Fig. 1B, left). Second, we built a simplified quasi-processive model, taking the crowding factor $(c)$ and processivity $(p)$ into consideration; $c$ was defined as a dimension-less value that compensates for the increase in or decrease of reaction rates under macromolecular crowding in comparison with the reaction rates in the ideal-dilute solution. $c$ reflects the effects of both thermodynamic activity and viscosity on the reaction rate $^{18}$. Therefore, the rate constants in all reactions should be multiplied by $c$ under a crowding condition (Fig. 1B, right).

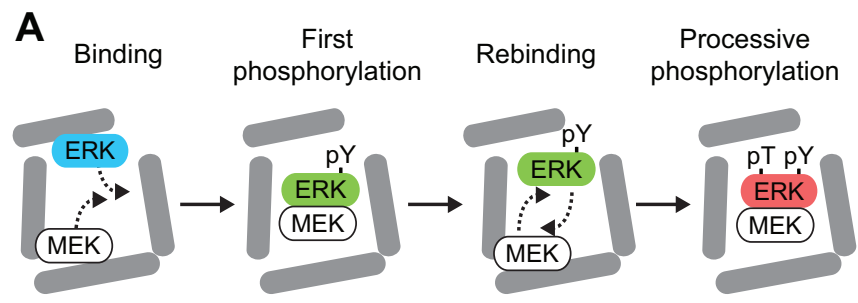

B Without molecular crowding

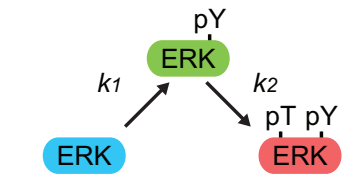

With molecular crowding

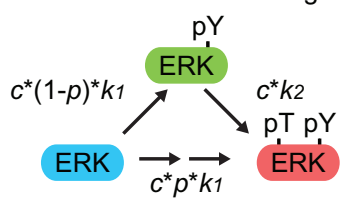

k1, k2: phosphorylation rate

$c$ : Crowding factor

$p$ : Processivity $(0<p<1)$

Figure $1 \mid$ Quasi-processive phosphorylation model under the condition of macromolecular crowding. (A) MEK (white) binds to and phosphorylates np-ERK (blue) at the tyrosine residue in the activation loop, and then dissociates from the product, pY-ERK (green). Under the condition of macromolecular crowding, the diffusions of both MEK and pY-ERK are restricted by the cage effect of the crowder molecules (grey). Thus, MEK rebinds to and phosphorylates pY-ERK with high probability. Consequently, MEK phosphorylates ERK in a processive manner, and produces pTpY-ERK (red). (B) The distributive (left) and quasi-processive phosphorylation model (right) are represented with parameters, including the phosphorylation rate constants, $k_{1}$ and $k_{2}$, crowding factor, $c$, and processivity, $p$. $p$ is defined as the probability of a processive reaction; i.e., the probability that pTpY-ERK will be generated directly from np-ERK (Fig. 1B, right). The second reaction of processive phosphorylation would be on the order of 10-0.1 $\mathrm{msec}^{23}$, and much faster than $k_{1}$ and $k_{2}$. For this reason, the reaction rate of the processive reaction can be described as $k_{1}^{*} p$. According to this definition, the rate constant of pY-ERK generation must be multiplied by $(1-p)$. With the $k_{1}$ and $k_{2}$ values determined in the absence of a crowder, $c$ and $p$ values can be obtained by fitting the experimental data with the quasi-processive model $^{24}$.

Measurements of crowding factor and processivity by in vitro ERK phosphorylation in the absence or presence of a crowder, poly-ethylene glycol 6000 . To evaluate the effect of macromolecular crowding on $c$ and $p$, the kinetics of ERK phosphorylation in vitro were studied in the absence or presence of a crowder agent, poly-ethylene glycol 6000 (PEG-6000) $^{24}$. A constitutively active mutant of MEK1 (MEK) and a kinase-dead mutant of GST-fused ERK2 (GST-ERK) were used as the kinase and substrate of the in vitro phosphorylation reaction, respectively. Samples were subjected to phospho-affinity gel electrophoresis with Phos-tag compound, which clearly separates four phospho-isoforms of ERK (Fig. 2A). The fractions of phospho-isoforms of ERK, namely, non-phosphorylated ERK (np-ERK), pY-ERK and pTpY-ERK, were quantified by an Odyssey Infra-red Imaging System (Fig. 2B, and Supplementary Fig. S1 online). Under our experimental condition, the order of electrophoretic mobility in the Phos-tag gel was, from the slowest to fastest, pY-ERK, pTpY-ERK, pT-ERK (if any), and np-ERK ${ }^{25,26}$. Two phosphorylation rates, $k_{1}$ and $k_{2}$, were determined from the data obtained in the absence of PEG-6000 (Fig. 2B, left). $c$ and $p$ at each concentration of PEG-6000 were determined by fitting the experimental data with the simplified quasi-processive model (Fig. 1B, right and Supplementary Fig. S1 and Dataset online).

PEG-6000 showed a dose-dependent biphasic effect on $c$ with the highest acceleration at approximately $10 \%$ (v/v) (Fig. 2C, green dots). This result is in agreement with the scheme documented by Minton et al. $^{18}$. $p$ increased steeply with increasing PEG concentration (Fig. 2C, red dots).

Formulation of the crowding effect on the overall reaction rate. We first formulated the effect of crowding on the overall reaction rates, namely, $c$ in this study. Minton and coworkers have established a basis for the mathematical framework of macromolecular crowding. Here, a biphasic effect on the enzymatic reaction rate is explained by increasing excluded volume and viscosity ${ }^{18}$. To formulate them, a simple enzymatic reaction is considered:

$$
E+S \longleftarrow E S \longrightarrow E+P,
$$

where $E, S, P$ and $E S$ represent the enzyme, substrate, product and enzyme-substrate transition state complex.

When the encounter rate between $E$ and $S$ is much higher than the conversion rate of $E S$ to $E+P$, the reaction is said to be transitionstate-limited. Under such a condition, the overall rate constant, $k_{f}$, is obtained by

$$
\frac{d[P]}{d t}=k_{f}=k_{t s}=k_{t s}^{0} \Gamma
$$

where $k_{t s}$ is the transition-state-limited rate constant, and $k_{t s}^{0}$ is the limiting value of $k_{t s}$ in the absence of an added $\operatorname{crowder}^{13,27} . \Gamma$ is defined by

$$
\Gamma=\frac{\gamma_{E} \gamma_{S}}{\gamma_{E S}}
$$

where $\gamma_{E}, \gamma_{S}$, and $\gamma_{E S}$ are the activity coefficients of the enzyme, substrate and enzyme-substrate transition state complex, respectively ${ }^{27}$. 

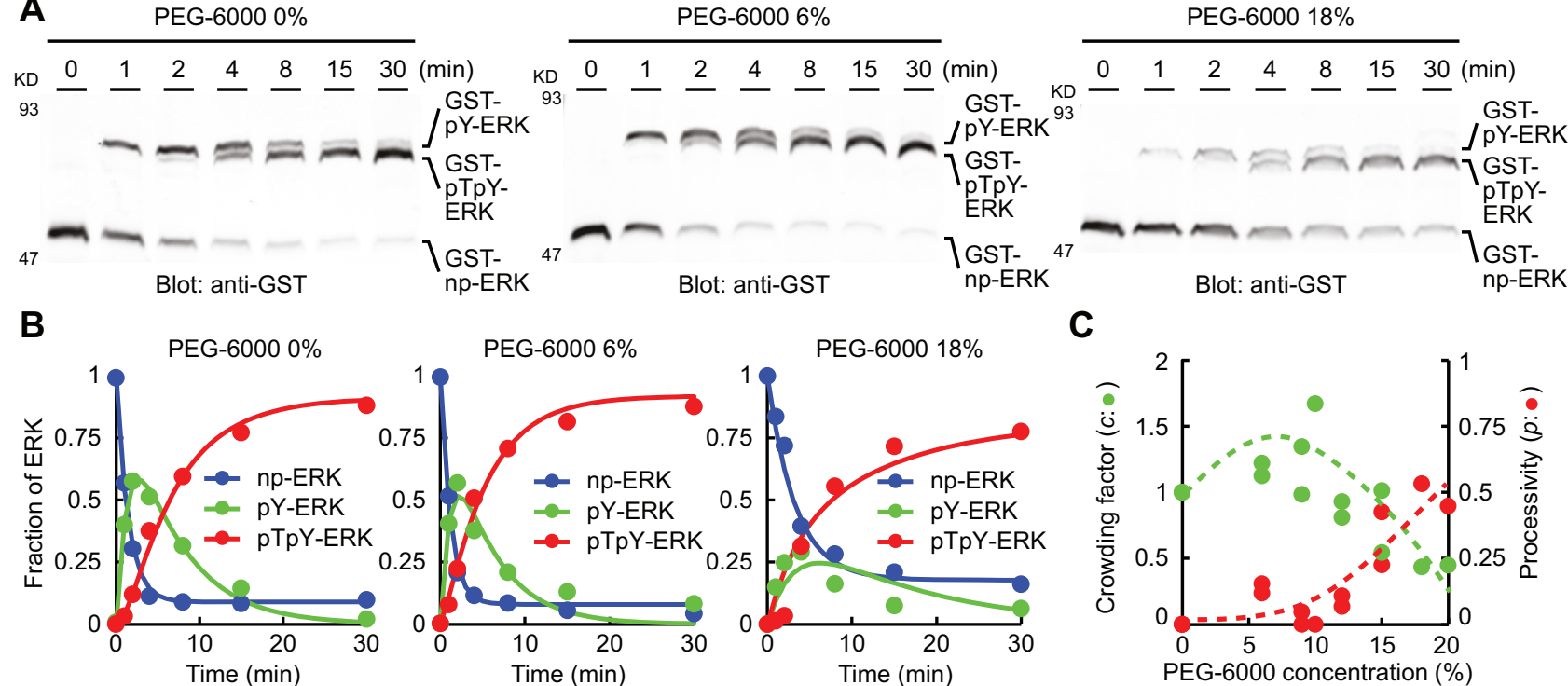

Blot: anti-GST

C

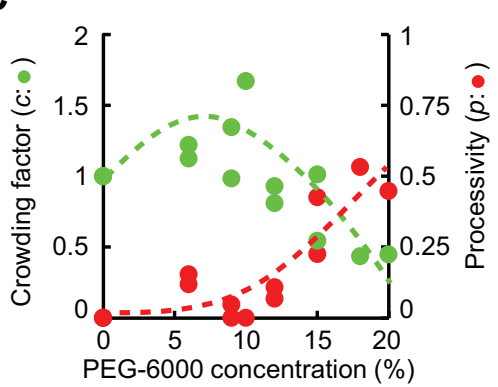

Figure $2 \mid$ In vitro ERK phosphorylation by MEK in the presence of PEG-6000 used as a crowder agent. (A) ERK was phosphorylated by MEK at the indicated concentration of PEG-6000 (v/v). The samples were subjected to Western blotting analysis with Phos-tag. (B) The fractions of phosphoisoforms of ERK determined in (A) are plotted against time. Lines represent the fractions of phospho-isoforms of ERK obtained by fitting the experimental data with the quasi-processive model in Fig. 1B. Raw and fitted data correspond to Exp \#2 in Supplementary Dataset. (C) The crowding factor ( $c$, green) and processivity ( $p$, red) at each PEG-6000 concentration were determined by fitting as described in the text. Additional data are included in Supplementary Figure S1. Dashed lines are drawn for emphasis.

The crowder and enzyme are considered as hard spherical particles with radius $r_{C}$, and $r_{E}$. The activity coefficient of the enzyme in a fluid of the crowder occupying fraction $\varphi$ of total fluid volume is given by

$$
\ln \gamma_{E}=-\ln (1-\varphi)+A_{1} Q+A_{2} Q^{2}+A_{3} Q^{3},
$$

where

$$
\begin{aligned}
& Q=\varphi /(1-\varphi), \\
& A_{1}=R^{3}+4.5 R^{2}+6 R+1.5, \\
& A_{2}=3 R^{3}+9 R^{2}+4.5 R, \\
& A_{3}=3 R^{3}+4.5 R^{2},
\end{aligned}
$$

and

$$
R \equiv r_{E} / r_{C}
$$

Similarly, the activity coefficients of the substrate and enzyme-substrate complex, $\gamma_{S}$ and $\gamma_{E S}$, are also obtained with their radii, $r_{S}$ and $r_{E S}$. We calculated the $\Gamma$ value in the presence of crowder agents of different sizes, $5 \mathrm{kD}, 20 \mathrm{kD}$, and $100 \mathrm{kD}$ (Fig. 3A). For this calculation, we assumed that all molecules were rigid spherical particles, in which radii were proportional to the cube root of their molecular weights. Consistent with previous reports ${ }^{27}$, the $\Gamma$ value increased monotonously with increasing volume fraction of crowder agent and correlated inversely with the molecular weight of the crowder ${ }^{17}$.

When the encounter rate between the enzyme and substrate is small relative to the conversion rate of enzyme-substrate complex to product, the reaction is said to be diffusion-limited. Under this condition, the encounter rate, $k_{e n c}$, which is proportional to the sum of the diffusion coefficients of enzyme and substrate, dictates the rate of reaction. The diffusion coefficient is inversely proportional to the viscosity of the solvent according to the Stokes-Einstein relationship. As an empirical approximation ${ }^{28,29}$, we may describe the rate of reaction with an exponential decay of crowder concentration

$$
k_{f}=k_{\text {enc }} \sim k_{\text {enc }}^{0} \exp (-g \varphi),
$$

where $k_{e n c}^{0}$ is the encounter rate constant in the absence of a volumeexcluding background species, and $g$ is a constant value that is a function of the relative sizes and shapes of the enzyme, substrate and crowder molecule ${ }^{27}$. We quantified the relative viscosity of PEG-6000 to water by an Ostwald viscometer, and obtained a $g$ value of 0.11 by fitting with the exponential function (Fig. 3B). Based on Eq. 6, the overall rate constant decreased exponentially with an increasing fraction of PEG volume under a diffusion-limited condition.

Eqs. 1 and 6 did not recapitulate the biphasic effect of the overall reaction rate independently of each other. To the best of our knowledge, the biphasic effects as observed in the experimental results have not been explicitly described by a mathematical framework, though such opposing effects of thermodynamic activity and viscosity on reaction rate have been well documented ${ }^{15,18,28}$. We considered an intermediate condition between a transition-state-limited reaction and a diffusion-limited reaction. Noyes ${ }^{30}$ indicates the relationship between the conversion and encounter rates as follows:

$$
\begin{aligned}
& k_{f}^{-1}=k_{t s}^{-1}+k_{e n c}^{-1}, \\
& k_{f}=\frac{k_{t s} k_{e n c}}{k_{t s}+k_{e n c}} .
\end{aligned}
$$

Combining Eqs. 1, 6 and 7 gives the following equation:

$$
k_{f}=\frac{k_{t s}^{0} k_{e n c}^{0} \Gamma \exp (-g \varphi)}{k_{t s}^{0} \Gamma+k_{e n c}^{0} \exp (-g \varphi)} .
$$

Using the overall reaction rate $k_{f}^{0}$ and ratio of the encounter rate to conversion rate $\theta$, Eq. 8 is written as

$$
k_{f}=\frac{(1+\theta) \Gamma \exp (-g \varphi)}{\Gamma+\theta \exp (-g \varphi)} k_{f}^{0},
$$

where

$$
k_{f}^{0}=\frac{k_{t s}^{0} k_{e n c}^{0}}{k_{t s}^{0}+k_{e n c}^{0}},
$$


A

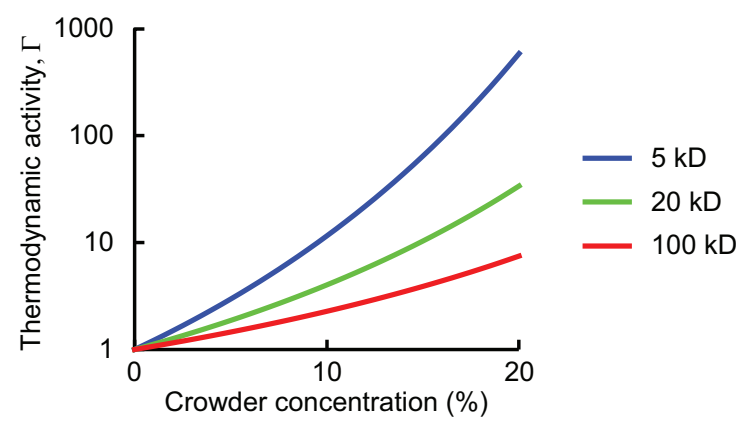

B

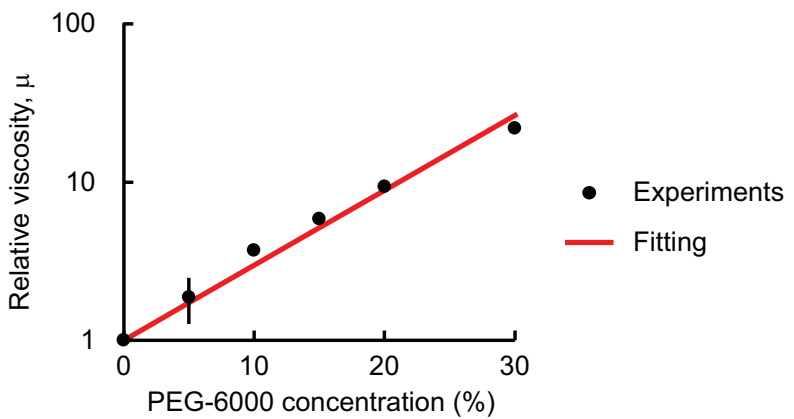

C

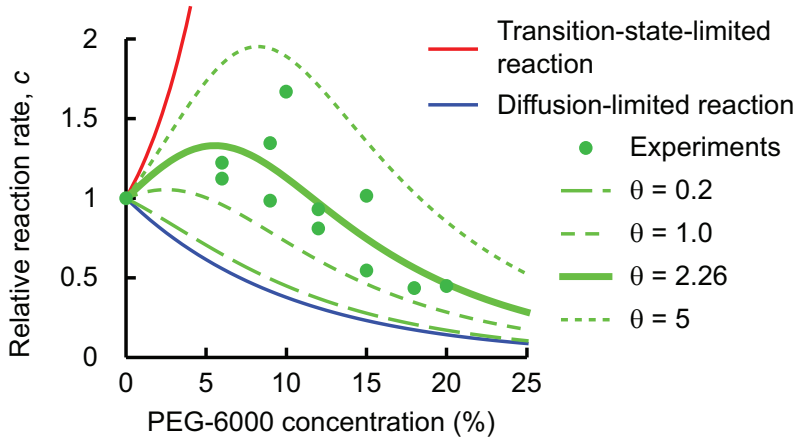

Figure $3 \mid$ Effects of macromolecular crowding on the overall reaction rate. (A) The $\Gamma$ value that represents the thermodynamic activity was numerically simulated, and semi-log plotted against the crowder concentration according to Eq. 2-5. The parameters of the numerical simulations were as follows: $r_{M E K}=45^{1 / 3}, r_{E R K}=42^{1 / 3}, r_{M E K-E R K}=87^{1 / 3}$, $r_{\text {croder }}=5^{1 / 3}$ (blue), $20^{1 / 3}$ (green), $100^{1 / 3}$ (red). (B) The relative viscosity of PEG-6000 to water was measured by an Ostwald viscometer, and semi-log plotted against the PEG-6000 concentration as dots with s.d. $(\mathrm{N}=3)$. The red line is fitted to the experimental data with a single exponential function (slope $=0.11$ ). (C) The crowding factor, $c$, was calculated according to Eq. 12 as a function of PEG-6000 concentration. Red and blue lines represent the effects of thermodynamic activity $(\theta \gg 0$ in Eq. 12) and viscosity $(\theta \ll 0)$ on the reaction rate, respectively. The green bold line is fitted to the experimental data (green dots as in Fig. 2C) with a $\theta$ value of 2.26. Relative reaction rates calculated at the indicated $\theta$ value are plotted as green dashed lines. The parameters of the numerical simulations are as follows: $r_{M E K}=45^{1 / 3}, r_{E R K}=42^{1 / 3}, r_{M E K-E R K}=87^{1 / 3}, r_{\text {croder }}=6^{1 / 3}, g=0.11$.

and

$$
\theta=\frac{k_{e n c}^{0}}{k_{t s}^{0}} .
$$

Based on the definition of $c$, we derived an equation of $c$ as follows:

$$
c=\frac{k_{f}}{k_{f}^{0}}=\frac{(1+\theta) \Gamma \exp (-g \varphi)}{\Gamma+\theta \exp (-g \varphi)}
$$

Importantly, under the transition-state-limited reaction $(\theta \gg 1)$, Eq. 9 corresponds to Eq. 1, whereas under the diffusion-limited reaction $(\theta \ll 1)$ Eq. 9 gives Eq. 6 . According to the value of $\Gamma$ and the $g$ value in PEG-6000 solution, we calculated the $c$ value at different concentrations of the crowder with a changing $\theta$ value as a variable (Fig. 3C, green lines). These results provided reasonable fits to the effect of crowder on the overall reaction rate in both the transition-statelimited and diffusion-limited reaction. By fitting the experimental data shown in Fig. $2 \mathrm{C}$ to this model, we obtained a $\theta$ value of 2.26 (Fig. 3C, bold green line). The value implicated the transition-statelimited reaction to some extent in ERK phosphorylation by MEK.

Formulation of processivity. We have defined the processivity as a probability of two-step successive phosphorylation reactions from np-ERK to pY-ERK to pTpY-ERK by an identical MEK. To formulate the processivity, we consider the situation where ERK has just been phosphorylated by MEK and converted to pY-ERK. Particles of MEK and pY-ERK are treated as point-like particles. These particles diffuse normally at all times in this model. Since MEK contains only a single ATP-binding pocket ${ }^{31}$, after the phosphorylation reaction, MEK must release ADP and bind to ATP in a manner dependent on an ordered bi-bi reaction. We define the period required for the replacement of ADP with ATP as a relaxation time, $\tau_{\text {rel }}$. The three-dimensional probability distribution of pY-ERK after $\tau_{r e l}$ is then given by

$$
\operatorname{pYERK}\left(r, \tau_{r e l}\right)=\frac{1}{\left(\sqrt{4 \pi D \tau_{r e l}}\right)^{3}} \exp \left(-\frac{r^{2}}{4 D \tau_{r e l}}\right),
$$

where $r$ is the distance from MEK and $D$ is the sum of the diffusion coefficient of MEK and pY-ERK in crowder solvent (Fig. 4A). We assume a reaction radius, $r_{\text {reac }}$, in which MEK rebinds to and
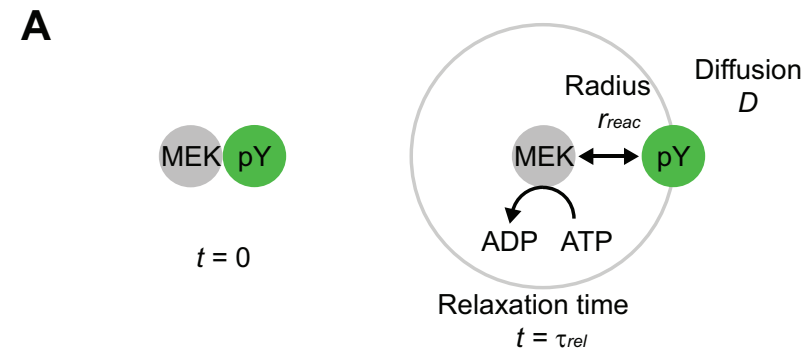

B

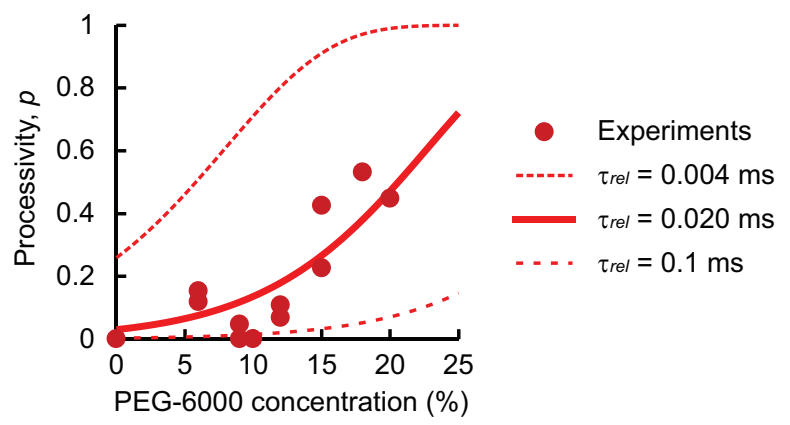

Figure $4 \mid$ Effect of macromolecular crowding on processivity.

(A) Schematic view of the processive phosphorylation. (Left) MEK phosphorylates the tyrosine residue of ERK at $t=0$. (Right) The relaxation time, $\tau_{\text {rel }}$ is defined as the period during which MEK releases ADP and binds to ATP. pY-ERK dissociated from MEK moves away by the diffusion coefficient of $D$. At $\mathrm{t}=\tau_{\text {rel }}$, MEK processively phosphorylates PY-ERK, if pY-ERK is located within the distance of $r_{\text {reao }}$ the reaction radius.

(B) According to Eq. 20, processivity was calculated as a function of PEG-6000 concentration. The red bold line is fitted to the experimental date (red dots as in Fig. 2C) with a $\tau_{r e l}$ value of 0.020 msec. Processivity values calculated at the indicated $\tau_{\text {rel }}$ values are depicted as red dashed lines. The parameters of the numerical simulations are as follows: $r_{\text {reac }}=10 \mathrm{~nm}$, $D_{\text {water }}=10 \mu \mathrm{m}^{2} / \mathrm{sec}, g=0.11$. 
processively phosphorylates pY-ERK within a time of $\tau_{r e l}$. The processivity, $p$, is then given by

$$
\begin{array}{r}
p\left(D, r_{\text {reac }}, \tau_{\text {rel }}\right)=\int_{0}^{2 \pi} \int_{0}^{\pi} \int_{0}^{r_{\text {reac }}} p \operatorname{HERK}\left(D, r, \tau_{\text {rel }}\right) \cdot r^{2} \sin \theta d \phi d \theta d r, \\
p\left(D, r_{\text {reac }}, \tau_{\text {rel }}\right)=\operatorname{Erf}\left(\frac{r_{\text {reac }}}{2 \sqrt{D \tau_{\text {rel }}}}\right)-\frac{r_{\text {reac }}}{\sqrt{\pi D \tau_{\text {rel }}}} \exp \left(-\frac{r_{\text {reac }}^{2}}{4 D \tau_{\text {rel }}}\right),
\end{array}
$$

where $\operatorname{Erf}$ is an error function given by

$$
\operatorname{Erf}(x)=\frac{2}{\sqrt{\pi}} \int_{0}^{x} \exp \left(-s^{2}\right) d s
$$

Taylor series of an error function and exponential function are given by

$$
\begin{gathered}
\operatorname{Erf}(x)=\frac{2}{\sqrt{\pi}}\left(x-\frac{x^{3}}{3}+\frac{x^{5}}{10}-\frac{x^{7}}{42}+\cdots\right) \\
\exp (x)=\sum_{n=0}^{\infty} \frac{x^{n}}{n !} .
\end{gathered}
$$

Based on Eqs. 17 and 18 for small $x \ll 1$, the Eq. 15 becomes

$$
p\left(D, r_{\text {reac }}, \tau_{r e l}\right) \approx \frac{r_{\text {reac }}^{3}}{4 \sqrt{\pi D^{3} \tau_{r e l}^{3}}} \propto r_{\text {reac }}^{3}, \tau_{r e l}^{-3 / 2}, D^{-3 / 2} .
$$

The relations in Eq. 19 were consistent with a part of the results reported previously ${ }^{23}$; the rebinding dynamics of MEK and pYERK is derived from a three-dimensional random walker returning to the origins. Because we simplified MEK and pY-ERK as point sources, we could not obtain the $t^{-1 / 2}$ decay dynamics of processivity in a range of times shorter than the time to travel a molecular diameter $^{23}$. The diffusion coefficient is inversely proportional to the viscosity of the solvent according to the Stokes-Einstein relationship. Thus, Eq. 15 becomes

$$
\begin{aligned}
p\left(D_{\text {water }}, r_{\text {reac }}, \tau_{\text {rel }}, \varphi\right)= & \operatorname{Erf}\left(\frac{r_{\text {reac }}}{2} \sqrt{\frac{\exp (g \varphi)}{D_{\text {water }} \tau_{\text {rel }}}}\right) \\
& -r_{\text {reac }} \sqrt{\frac{\exp (g \varphi)}{\pi D_{\text {water }} \tau_{\text {rel }}}} \exp \left(-\frac{r_{\text {reac }}^{2} \exp (g \varphi)}{4 D_{\text {water }} \tau_{\text {rel }}}\right),
\end{aligned}
$$

where $D_{\text {water }}$ is the sum of the diffusion coefficient of MEK and pYERK in water. To evaluate this model, we calculated processivity according to Eq. 20. $D_{\text {water }}$ was set at $10 \mu \mathrm{m}^{2} / \mathrm{sec}$ based on the viscosity of water and the size of MEK and ERK. In this model, we did not consider the effect of viscosity on diffusion coefficient of ADP/ ATP, i.e. $\tau_{\text {rel }}$, because of their high concentration and fast diffusion (see Discussion). We set the reaction radius $r_{\text {reac }}$ at $10 \mathrm{~nm}$, which was comparable to the sum of the radius of MEK and ERK molecules. Processivity was obtained by changing the value of $\tau_{r e l}$, namely, the reactivation time of MEK, and plotted against the concentration of PEG-6000, which increased viscosity (Fig. 4B). The processivity elevated with increasing concentration of PEG-6000. Furthermore, the model demonstrated a best fit to the experimental data at the $\tau_{r e l}$ value of $0.020 \mathrm{msec}$ (Fig. 4B, bold line).

A full reaction kinetics model of ERK phosphorylation. In the quasi-processive phosphorylation model described in this study, the association and dissociation of ERK to MEK were neglected for the simplicity. Therefore, we performed similar analyses based on the full reaction kinetics of ERK phosphorylation model. Kinetic parameters were fitted with experimental data in the absence of PEG6000 (Fig. S2A, left), followed by the fitting of crowding factor, $c$, and dissociation constant, $k_{b-}$, in the full model with experimental data under the condition of PEG-6000 (Fig. S2A right). Of note, the reaction model can strictly define processivity as $k_{2} /\left(k_{b-}+k_{2}\right)$ without any need to introduce an empirical parameter of processivity in the simplified model (Fig. 1B). The full model recapitulated time course of ERK phosphorylation in the absence or presence of PEG6000 (Fig. S2B). The fitted values of crowding factor (Fig. S2C) and processivity (Fig. S2D) provided consistent values of $\theta(1.80)$ and $\tau_{\text {rel }}(0.024 \mathrm{msec})$ in Eq. 12 and Eq. 20 , respectively.

Analysis of crowding factor and processivity based on the scaled particle theory. The crowding effect (Eq. 12) and processivity (Eq. 20) explicitly include viscosity, which is macroscopic parameter described by the Stokes-Einstein law. Therefore, we next examined the microscopic effect of crowder on crowding factor and processivity by estimating diffusion coefficients based on the scaled particle theory $(\mathrm{SPT})^{32}$. Let $r_{t}$ represent the radius of the tracer species, $\Delta r^{\prime}$ the approximated length of the Brownian displacement ${ }^{32}$. The scaled particle theory yields

$$
\begin{aligned}
\ln \left(\mathrm{D} / \mathrm{D}_{\text {water }}\right)= & -\Delta r^{\prime} \\
& {\left[A_{1}+A_{2}\left(2 r_{t}+\Delta r^{\prime}\right)+A_{3}\left(3 r_{t}^{2}+3 r_{t} \Delta r^{\prime}+\Delta r^{\prime 2}\right)\right], }
\end{aligned}
$$

where $D$ and $D_{\text {water }}$ are the diffusion coefficient of tracer species in the presence or absence of background crowder. $A_{i}$ is given by

$$
\begin{gathered}
A_{0}=-\ln \left(1-S_{3}\right), \\
A_{1}=6 S_{2} /\left(1-S_{3}\right), \\
A_{2}=\left[12 S_{1} /\left(1-S_{3}\right)\right]+\left[18 S_{2}^{2} /\left(1-S_{3}\right)^{2}\right], \\
A_{3}=\left[8 S_{0} /\left(1-S_{3}\right)\right]+\left[24 S_{1} S_{2} /\left(1-S_{3}\right)^{2}\right]+\left[24 S_{2}^{3} /\left(1-S_{3}\right)^{3}\right], \\
S_{j}=\frac{\pi}{6} v\left(2 r_{b}\right)^{j}, \\
v=\frac{c_{b a c k} N_{a}}{1000 M},
\end{gathered}
$$

where $r_{b}$ is radius of background molecule, $M$ is molecular weight, $N_{a}$ is Avogadro's number, $c_{b a c k}$ is a concentration of background molecules [g/litter], and $v$ is in $\mathrm{cm}^{-3}$. We obtained almost same result of BSA self-diffusion as previously reported in Muramatsu and Minton, indicating the valid calculation (Fig. S3A). Next, logarithmic ratio of $D$ to $D_{\text {water }}$ of relative diffusion coefficient of MEK and ERK with the increase in PEG-6000 concentration was estimated by SPT (Fig. S3B). The microscopic model with SPT significantly underestimated the relative diffusion coefficient in the presence of PEG6000 (Fig. S3B, red line) in comparison to the relative diffusion coefficients calculated by the experimental data with Ostwald viscometer (Fig. S3B, blue line). We recalculated and fitted the crowding factor (Fig. S3C) and processivity (Fig. S3D) using SPT with the experimental data; however there existed significant discrepancy between the experimental data and mathematical model with the scaled particle theory. This could be due to steeper reduction of the relative diffusion coefficient between MEK and ERK obtained by SPT. From these results, we concluded, at this time, that the increase in viscosity under the condition of macromolecular crowding was the responsible factor for the crowding factor and processivity. The clear dissection of difference between microscopic and macroscopic effect of molecular crowding is one of challenging tasks for future studies. Simulation of the enzymatic reaction at the particle level will enable to directly address this issue ${ }^{23}$.

Analytical solution of the simplified quasi-processive phosphorylation model. Finally, based on Eq. 12 and 20, analytical solutions of np-ERK, pY-ERK and pTpY-ERK concentration, [npERK], [pYE- 
$R K]$, and [pTpYERK], respectively, in the quasi-processive model (Fig. 1B) are given by

$$
\begin{gathered}
{[n p E R K](t)=E R K_{0} \cdot e^{-c \cdot k_{1} t}} \\
{[p Y E R K](t)=E R K_{0} \frac{e^{-c\left(k_{1}+k_{2}\right) t}\left(e^{c \cdot k_{2} t}-e^{c \cdot k_{1} t}\right) k_{1}(p-1)}{k_{1}-k_{2}}}
\end{gathered}
$$

$$
\begin{aligned}
& {[\text { PTpYERK }](t)=E R K_{0}} \\
& \frac{e^{-c\left(k_{1}+k_{2}\right) t}\left\{e^{c\left(k_{1}+k_{2}\right) t}\left(k_{1}+k_{2}\right)+e^{c \cdot k_{1} t} k_{1}(p-1)+e^{c \cdot k_{2} t}\left(k_{2}-k_{1} p\right)\right\}}{k_{1}-k_{2}}
\end{aligned}
$$

where the $[n p E R K]$, $[p Y E R K]$, and $[p T p Y E R K]$ at time $=0$ are $E K_{0}$, 0 , and 0 , respectively. $k_{1}$ and $k_{2}$ were first-order successive phosphorylation rates [/sec] of ERK by MEK based on our previous study ${ }^{24}$. To simplify these equations, we omitted the factor of MEK concentration, and included it implicitly in $k_{1}$ and $k_{2}$. In general, analytical solutions of two successive reactions were obtained at $c=1$ and $p=0$ as follows:

$$
\begin{gathered}
{[n p E R K](t)=E R K_{0} \cdot e^{-k_{1} t}} \\
{[p Y E R K](t)=E R K_{0} \frac{e^{-\left(k_{1}+k_{2}\right) t}\left(-e^{k_{2} t}+e^{k_{1} t}\right) k_{1}}{k_{1}-k_{2}}}
\end{gathered}
$$

$[p T p Y E R K](t)=E R K_{0}$

$$
\frac{e^{-\left(k_{1}+k_{2}\right) t}\left\{e^{\left(k_{1}+k_{2}\right) t}\left(k_{1}+k_{2}\right)-e^{k_{1} t} k_{1}+k_{2} e^{k_{2} t}\right\}}{k_{1}-k_{2}}
$$

In the quasi-processive phosphorylation model, we assumed that the reaction rate of processive phosphorylation is equivalent to $k_{1}^{*} p$, because the second reaction of processive phosphorylation would be much faster than $k_{1}$ and $k_{2}$. These equations provided a good agreement with the dynamics of ERK phosphorylation at different
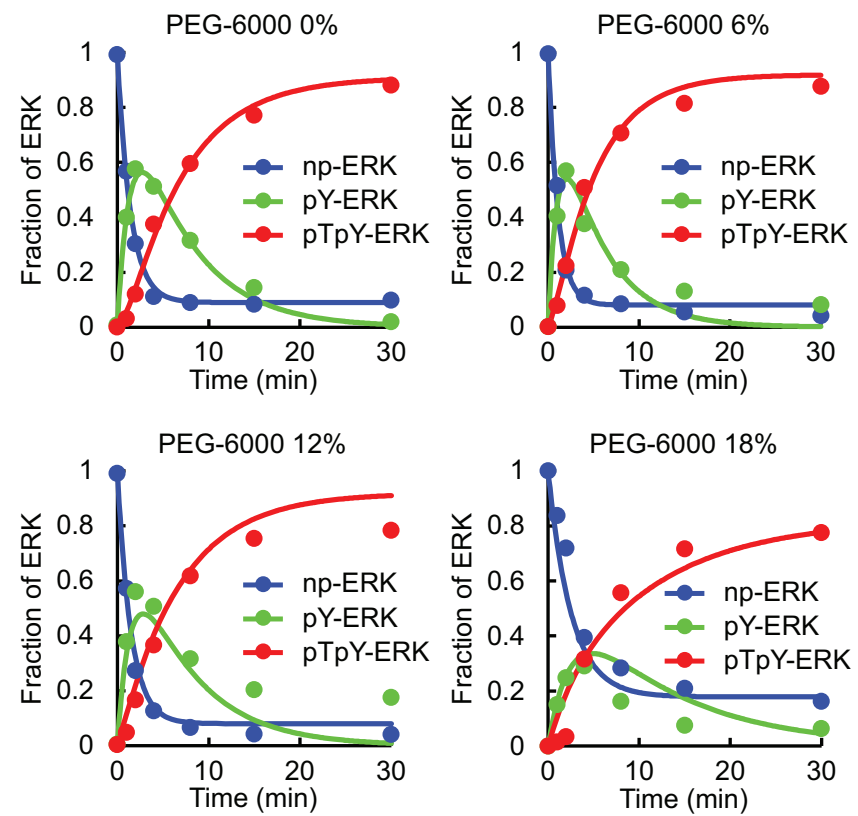

Figure 5 | Analytical solution of ERK phosphorylation by MEK under the condition of macromolecular crowding. The fractions of np-ERK (blue), pY-ERK (green) and pTpY-ERK (red) were calculated according to Eq. 21-23. Experimental data are the same in Figure 2A and 2B (Exp. \#2 in Supplementary Data set). The parameters of the numerical simulations were as follows: $r_{M E K}=45^{1 / 3}, r_{E R K}=42^{1 / 3}, r_{M E K-E R K}=87^{1 / 3}, r_{\text {croder }}=6^{1 / 3}$, $g=0.11, \theta=2.26, r_{\text {reac }}=10 \mathrm{~nm}, D_{\text {water }}=10 \mu \mathrm{m}^{2} / \mathrm{sec}, \tau_{\text {rel }}=0.020 \mathrm{msec}$. concentrations of PEG-6000 (Fig. 5), supporting the validity of the model.

\section{Discussion}

In this study, we provided for the first time the mathematical framework for the rate constant and processivity at intermediate conditions between transition-state-limited and diffusion-limited reactions under the condition of molecular crowding. Moreover, we derived an analytical expression for the kinetics in ERK processive phosphorylation, demonstrating time courses of phosphorylation as a function of crowder concentration, $\phi$. These models recapitulated reaction kinetics of dual phosphorylation of ERK by MEK with or without crowder agent. However, slight, but not ignorable, disagreement is also evident between the experimental data and the model, especially in late phase of higher concentrations of PEG-6000, 12\% and $18 \%$. This result suggests that the PEG-6000 solution demonstrates a spatially heterogeneous environment, which entails the introduction of a time-dependent rate coefficient with a fractal kinetics mode ${ }^{13,33}$. In a previous simulation study, diffusion obstacles gave rise to anomalous diffusion ${ }^{34}$. Consistent with this idea, anomalous diffusion in the cytoplasm was also observed by fluorescence correlation spectroscopy $y^{35-37}$. Moreover, it has recently been indicated that fractal structures exist in the nucleus ${ }^{38,39}$. These findings should pave the way for future work that will improve the model more quantitatively.

We demonstrated a clear dependency of processivity in Eq. 19 on diffusion coefficient, $D$, reactivation time, $\tau_{\text {rel }}$, and reactivation radius, $r_{\text {reac }}$. Membrane-anchoring or transmembrane proteins are well-known to show slower diffusion coefficients in comparison to those of cytoplasmic proteins ${ }^{40}$. Of note, many of receptor tyrosine kinases (RTKs) such as epidermal growth factor receptor form homo- and hetero-dimer upon ligand stimulation on membrane, and transphosphorylates multiple sites of tyrosine residues in $\mathrm{RTKs}^{41}$, strongly suggesting the possibility of processive phosphorylation in the RTK activation. Diffusion-controlled ligand-receptor kinetics supports this possibility ${ }^{42}$. Furthermore, rebinding event would take place in protein translocation on nucleic acid $^{43,44}$. Intriguingly, by analogy to the diffusion coefficient, clustering or confinement of molecules in small compartment also induced the increase in processivity ${ }^{45}$.

Taking the high concentration and fast diffusion rate of intracellular ATP molecules into consideration, the rate-limiting step of $\mathrm{ADP} / \mathrm{ATP}$ exchange, i.e. reactivation time, $\tau_{\text {rel }}$, is likely to be determined by the ADP release from MEK. The dissociation rate constants of ADP from kinases have been reported to vary over a wide range with an order of magnitude of 3 to $4\left(0.23 \mathrm{sec}^{-1}\right.$ to $\left.140 \mathrm{sec}^{-1}\right)^{46-50}$. The ADP/ATP exchange rate predicted in this study, $50000 \mathrm{sec}^{-1}$, which is the reciprocal of $\tau_{r e l}$ is much larger than the dissociation rate constants reported previously. There are at least two possible explanations for this discrepancy. First, the reaction radius, $r_{\text {reac }}$ may have been underestimated in this study. Currently, we do not have any methods to quantitatively estimate the reaction radius. Second, the phosphorylation reaction model may not be correct. We employed an ordered bi-bi sequential reaction of processive phosphorylation, in which MEK dissociated from pY-ERK, followed by exchange of ADP with ATP to phosphorylate PY-ERK. If MEK processively phosphorylates ERK through a random bi-bi mechanism, the processivity will increase even under a slow ADP/ATP exchange rate. In fact, Horiuchi et al. have indicated that the mechanism of MEK turnover involves random addition of substrate and $\mathrm{ATP}^{51}$. Although they have also suggested the ordered release of product from $\mathrm{MEK}^{51}$, effects of crowding on the mechanism of MEK turnover have not been fully characterized, and should be considered in a future study.

The mathematical models developed here integrate three effects of molecular crowding on the reaction rate-namely, thermodynamic activity, viscosity and processivity. These models were consistent 
with experimental data supporting the processive ERK phosphorylation by MEK. Our findings will contribute to the effort to quantitatively simulate intracellular signal transduction.

\section{Methods}

Plasmids. pGEX4T3-ERK2KR, a kinase-deficient mutant (K57R) of Xenopus ERK2, and pET-12HisFLAG-MEK1SDSE, a constitutively active mutant (S218D/S222E) of Xenopus MEK1, have been purified as described previously ${ }^{24}$.

Reagents and antibodies. Phos-tag acrylamide was obtained from the Phos-tag Consortium (http://www.phos-tag.com/). Fifty percent polyethylene glycol 6000 (PEG-6000) solution was purchased from Hampton Research (Aliso Viejo, CA). Anti-ERK1/2 and anti-phospho-ERK1/2 (Thr202/Tyr204 and Thr185/Tyr187, respectively) were purchased from Cell Signaling Technology (Beverly, MA). LI-COR-blocking buffer and IRDye680- and IRDye800-conjugated anti-rabbit and anti-mouse IgG secondary antibodies were obtained from LI-COR Bioscience (Lincoln, NE).

Purification of recombinant proteins and in vitro kinase assay. GST-ERK2KR and 12His-MEK1SDSE were prepared as described previously ${ }^{24}$. For the in vitro kinase assay, $1 \mu \mathrm{M}$ GST-ERK2, $0.1 \mu \mathrm{M}$ 12HisFLAG-MEK1SDSE and different concentrations of PEG-6000 $(0 \sim 20 \% \mathrm{v} / \mathrm{v})$ were incubated in in vitro kinase buffer (50 mM Tris [pH 7.5], $10 \mathrm{mM} \mathrm{MgCl}, 0.02 \%$ BSA $0.2 \mathrm{mM}$ DTT). The reaction was started by adding $5 \times$ ATP solution $\left(5 \mathrm{mM}\right.$ ATP, $50 \mathrm{mM} \mathrm{MgCl}_{2}, 25 \mathrm{mM}$ Tris [pH 7.2], $0.15 \mathrm{M} \mathrm{NaCl}$ ) and stopped at the indicated time point by adding SDS sample buffer.

Phos-tag polyacrylamide gel electrophoresis. Phos-tag polyacrylamide gel electrophoresis was performed essentially as described previously $y^{24,25}$. Briefly, $50 \mu \mathrm{M}$ Phos-tag and $100 \mu \mathrm{M} \mathrm{MnCl}_{2}$ were added to conventional SDS-polyacrylamide separation gels according to the manufacturer's protocol, respectively. Proteins were detected by using an Odyssey Infrared Imaging System (LI-COR).

Measurements of viscosity. Viscosities of the aqueous PEG-6000 solutions relative to water were determined by using an Ostwald viscometer at $37^{\circ} \mathrm{C}$ in a water bath.

Parameter search. Numerical simulation and parameter search were implemented by MATLAB software with ode15s function and fminsearch function, respectively.

1. Hanahan, D. \& Weinberg Robert, A. Hallmarks of Cancer: The Next Generation. Cell 144, 646-674 (2011)

2. Kholodenko, B. N. Cell-signalling dynamics in time and space. Nat. Rev. Mol. Cell Biol. 7, 165-176 (2006)

3. Kitano, H., Funahashi, A., Matsuoka, Y. \& Oda, K. Using process diagrams for the graphical representation of biological networks. Nat. Biotechnol. 23, 961-966 (2005).

4. Schoeberl, B., Eichler-Jonsson, C., Gilles, E. D. \& Muller, G. Computational modeling of the dynamics of the MAP kinase cascade activated by surface and internalized EGF receptors. Nat. Biotechnol. 20, 370-375 (2002).

5. Fujioka, A. et al. Dynamics of the Ras/ERK MAPK cascade as monitored by fluorescent probes. J. Biol. Chem. 281, 8917-8926 (2006).

6. Matsunaga-Udagawa, R. et al. The scaffold protein Shoc2/SUR-8 accelerates the interaction of Ras and Raf. J Biol. Chem. 285, 7818-7826 (2010).

7. Kamioka, Y., Yasuda, S., Fujita, Y., Aoki, K. \& Matsuda, M. Multiple decisive phosphorylation sites for the negative feedback regulation of SOS1 via ERK. J Biol. Chem. 285, 33540-33548 (2010).

8. Aoki, K., Nakamura, T., Inoue, T., Meyer, T. \& Matsuda, M. An essential role for the SHIP2-dependent negative feedback loop in neuritogenesis of nerve growth factor-stimulated PC12 cells. J. Cell Biol. 177, 817-827 (2007).

9. Minton, A. P. How can biochemical reactions within cells differ from those in test tubes? J Cell Sci. 119, 2863-2869 (2006).

10. Medalia, O. et al. Macromolecular architecture in eukaryotic cells visualized by cryoelectron tomography. Science 298, 1209-1213 (2002).

11. Fulton, A. B. How crowded is the cytoplasm? Cell 30, 345-347 (1982),

12. Zimmerman, S. B. \& Trach, S. O. Estimation of macromolecule concentrations and excluded volume effects for the cytoplasm of Escherichia coli. J. Mol. Biol. 222, 599-620 (1991).

13. Schnell, S. \& Turner, T. E. Reaction kinetics in intracellular environments with macromolecular crowding: simulations and rate laws. Prog. Biophys. Mol. Biol. 85, 235-260 (2004).

14. Al-Habori, M. Macromolecular crowding and its role as intracellular signalling of cell volume regulation. Int. J. Biochem. Cell Biol. 33, 844-864 (2001).

15. Ellis, R. J. Macromolecular crowding: an important but neglected aspect of the intracellular environment. Curr. Opin. Struct. Biol. 11, 114-119 (2001).

16. Ellis, R. J. Macromolecular crowding: obvious but underappreciated. Trends Biochem. Sci. 26, 597-604 (2001).

17. Hall, D. \& Minton, A. P. Macromolecular crowding: qualitative and semiquantitative successes, quantitative challenges. Biochim. Biophys. Acta 1649, $127-139$ (2003).
18. Zimmerman, S. B. \& Minton, A. P. Macromolecular crowding: biochemical, biophysical, and physiological consequences. Annu. Rev. Biophys. Biomol. Struct. 22, 27-65 (1993).

19. Koivomagi, M. et al. Cascades of multisite phosphorylation control Sic1 destruction at the onset of S phase. Nature 480, 128-131 (2011).

20. Pellicena, P. \& Miller, W. T. Processive phosphorylation of p130Cas by Src depends on SH3-polyproline interactions. J. Biol. Chem. 276, 28190-28196 (2001).

21. Williamson, A. et al. Regulation of ubiquitin chain initiation to control the timing of substrate degradation. Mol. Cell 42, 744-757 (2011).

22. Patwardhan, P. \& Miller, W. T. Processive phosphorylation: mechanism and biological importance. Cell Signal. 19, 2218-2226 (2007).

23. Takahashi, K., Tanase-Nicola, S. \& ten Wolde, P. R. Spatio-temporal correlations can drastically change the response of a MAPK pathway. Proc. Natl. Acad. Sci. U. S. A. 107, 2473-2478 (2010)

24. Aoki, K., Yamada, M., Kunida, K., Yasuda, S. \& Matsuda, M. Processive phosphorylation of ERK MAP kinase in mammalian cells. Proc. Natl. Acad. Sci. U. S. A. 108, 12675-12680 (2011).

25. Kinoshita, E., Kinoshita-Kikuta, E., Takiyama, K. \& Koike, T. Phosphate-binding tag, a new tool to visualize phosphorylated proteins. Mol. Cell Proteomics. 5, 749-757 (2006).

26. Kinoshita, E., Kinoshita-Kikuta, E. \& Koike, T. Phos-tag SDS-PAGE systems for phosphorylation profiling of proteins with a wide range of molecular masses under neutral pH conditions. Proteomics 12, 192-202 (2012).

27. Minton, A. P. Molecular crowding: analysis of effects of high concentrations of inert cosolutes on biochemical equilibria and rates in terms of volume exclusion. Methods Enzymol. 295, 127-149 (1998).

28. Minton, A. P. Excluded Volume as a Determinant of Macromolecular Structure and Reactivity. Biopolymers 20, 2093-2120 (1981).

29. Caldin, E. F. \& Hasinoff, B. B. Diffusion-controlled kinetics in the reaction of ferroprotoporphyrin IX with carbon monoxide. Journal of the Chemical Society, Faraday Transactions 1: Physical Chemistry in Condensed Phases 71, 515-527 (1975).

30. Noyes, R. M. Effects of Diffusion Rates on Chemical Kinetics. Progress Reaction Kinetics. Peter, G. an editor. Pergamon Press, New York. 1, 129-160 (1961).

31. Fischmann, T. O. et al. Crystal structures of MEK1 binary and ternary complexes with nucleotides and inhibitors. Biochemistry (Mosc.) 48, 2661-2674 (2009).

32. Muramatsu, N. \& Minton, A. P. Tracer diffusion of globular proteins in concentrated protein solutions. Proc. Natl. Acad. Sci. U. S. A. 85, 2984-2988 (1988).

33. Kopelman, R., Parus, S. \& Prasad, J. Fractal-like exciton kinetics in porous glasses, organic membranes, and filter papers. Phys. Rev. Lett. 56, 1742-1745 (1986).

34. Saxton, M. J. Anomalous diffusion due to obstacles: a Monte Carlo study. Biophys. J. 66, 394-401 (1994)

35. Wachsmuth, M., Waldeck, W. \& Langowski, J. Anomalous diffusion of fluorescent probes inside living cell nuclei investigated by spatially-resolved fluorescence correlation spectroscopy. J. Mol. Biol. 298, 677-689 (2000).

36. Malchus, N. \& Weiss, M. Elucidating anomalous protein diffusion in living cells with fluorescence correlation spectroscopy-facts and pitfalls. J. Fluoresc. 20, 19-26 (2010).

37. Weiss, M., Hashimoto, H. \& Nilsson, T. Anomalous protein diffusion in living cells as seen by fluorescence correlation spectroscopy. Biophys. J. 84, 4043-4052 (2003).

38. Bancaud, A. et al. Molecular crowding affects diffusion and binding of nuclear proteins in heterochromatin and reveals the fractal organization of chromatin. EMBO J. 28, 3785-3798 (2009).

39. Lieberman-Aiden, E. et al. Comprehensive mapping of long-range interactions reveals folding principles of the human genome. Science 326, 289-293 (2009).

40. Reits, E. A. \& Neefjes, J. J. From fixed to FRAP: measuring protein mobility and activity in living cells. Nat. Cell Biol. 3, E145-147 (2001).

41. Jones, R. B., Gordus, A., Krall, J. A. \& MacBeath, G. A quantitative protein interaction network for the ErbB receptors using protein microarrays. Nature 439, 168-174 (2006).

42. Goldstein, B. \& Dembo, M. Approximating the effects of diffusion on reversible reactions at the cell surface: ligand-receptor kinetics. Biophys. J. 68, 1222-1230 (1995).

43. Zhou, H. X. A model for the mediation of processivity of DNA-targeting proteins by nonspecific binding: dependence on DNA length and presence of obstacles. Biophys. J. 88, 1608-1615 (2005).

44. Berg, O. G. \& von Hippel, P. H. Diffusion-controlled macromolecular interactions. Annu. Rev. Biophys. Biophys. Chem. 14, 131-160 (1985).

45. Mugler, A., Bailey, A. G., Takahashi, K. \& ten Wolde, P. R. Membrane clustering and the role of rebinding in biochemical signaling. Biophys. J. 102, 1069-1078 (2012).

46. Jan, A. Y., Johnson, E. F., Diamonti, A. J., Carraway, I. K. \& Anderson, K. S. Insights into the HER-2 receptor tyrosine kinase mechanism and substrate specificity using a transient kinetic analysis. Biochemistry (Mosc.) 39, 9786-9803 (2000).

47. Shaffer, J., Sun, G. \& Adams, J. A. Nucleotide release and associated conformational changes regulate function in the $\mathrm{COOH}$-terminal Src kinase, Csk. Biochemistry (Mosc.) 40, 11149-11155 (2001). 
48. Callaway, K., Waas, W. F., Rainey, M. A., Ren, P. \& Dalby, K. N. Phosphorylation of the transcription factor Ets-1 by ERK2: rapid dissociation of ADP and phospho-Ets-1. Biochemistry (Mosc.) 49, 3619-3630 (2010).

49. Shaffer, J. \& Adams, J. A. Detection of conformational changes along the kinetic pathway of protein kinase A using a catalytic trapping technique. Biochemistry (Mosc.) 38, 12072-12079 (1999).

50. Lew, J., Taylor, S. S. \& Adams, J. A. Identification of a partially rate-determining step in the catalytic mechanism of cAMP-dependent protein kinase: a transient kinetic study using stopped-flow fluorescence spectroscopy. Biochemistry (Mosc.) 36, 6717-6724 (1997).

51. Horiuchi, K. Y., Scherle, P. A., Trzaskos, J. M. \& Copeland, R. A. Competitive inhibition of MAP kinase activation by a peptide representing the alpha $\mathrm{C}$ helix of ERK. Biochemistry (Mosc.) 37, 8879-8885 (1998).

\section{Acknowledgements}

We thank the members of the Matsuda Laboratory for their helpful discussions. KA and MM were supported by Research Program of Innovative Cell Biology by Innovative
Technology (Cell Innovation) from the Ministry of Education, Culture, Sports, and Science, Japan. KA was supported by the JST PRESTO program and JSPS KAKENHI (21790273).

\section{Author contributions}

K.A., K.T., K.K. and M.M. designed research; K.A. performed experiments; K.A., K.T. and K.K. analyzed data; K.A., K.T., K.K. and M.M. wrote the paper.

\section{Additional information}

Supplementary information accompanies this paper at http://www.nature.com/ scientificreports

Competing financial interests: The authors declare no competing financial interests. License: This work is licensed under a Creative Commons Attribution-NonCommercial-NoDerivs 3.0 Unported License. To view a copy of this license, visit http://creativecommons.org/licenses/by-nc-nd/3.0/

How to cite this article: Aoki, K., Takahashi, K., Kaizu, K. \& Matsuda, M. A Quantitative Model of ERK MAP Kinase Phosphorylation in Crowded Media. Sci. Rep. 3, 1541; DOI:10.1038/srep01541 (2013). 